\title{
Pacific
}

Journal of

Mathematics

\section{AXIAL SYMMETRY AND REGULARITY OF SOLUTIONS TO AN INTEGRAL EQUATION IN A HALF-SPACE}

\author{
GUOZHEN LU AND JIUYI ZHU
}




\title{
AXIAL SYMMETRY AND REGULARITY OF SOLUTIONS TO AN INTEGRAL EQUATION IN A HALF-SPACE
}

\author{
GUOZHEN LU AND JIUYI ZHU
}

We consider the integral equation

$$
u(x)=\int_{\mathbb{R}_{+}^{n}} G(x, y) f(u(y)) d y,
$$

where $G(x, y)$ is the Green's function of the corresponding polyharmonic Dirichlet problem in a half-space. We prove by the method of moving planes in integral form that, under some integrability conditions, the solutions are axially symmetric with respect to some line parallel to the $x_{n}$-axis and nondecreasing in the $x_{n}$ direction, which further implies the nonexistence of solutions. We also show similar results for a class of systems of integral equations. This appears to be the first paper in which the moving plane method in integral form is employed in a half-space to derive axial symmetry.

We also obtain the regularity of the integral equation in a half-space

$$
u(x)=\int_{\mathbb{R}_{+}^{n}} G(x, y)|u(y)|^{p-1} u(y) d y
$$

by the regularity lifting method. As a corollary, we prove the nonexistence of nonnegative solutions to this equation. Moreover, we show that the nonnegative solutions in this equation only depend on $x_{n}$ if $u \in L_{\mathrm{loc}}^{2 n /(n-2 m)}\left(\mathbb{R}_{+}^{n}\right)$ and $1<p<(n+2 m) /(n-2 m)$.

\section{Introduction}

We study the properties of nonnegative solutions of the following integral equations in a half-space:

$$
u(x)=\int_{\mathbb{R}_{+}^{n}} G(x, y) f(u) d y,
$$

where $\mathbb{R}_{+}^{n}=\left\{x \in \mathbb{R}^{n} \mid x_{n}>0\right\}, n>2 m$ is the dimension of the half-space, and $G(x, y)$ is the Green's functions of $(-\Delta)^{m}$ related to a Dirichlet boundary condition in $\mathbb{R}_{+}^{n}$

This research is partially supported by NSF grant number DMS-0901761.

MSC2010: primary 35J60; secondary 45G15.

Keywords: axial symmetry, regularity of solutions, half-space, Green's functions for polyharmonic operators, integral equation, nonexistence of solutions. 
that will be presented in Section 2. We also assume that $f(u(x))$ satisfies $\left(f_{1}\right) f:[0, \infty) \rightarrow \mathbb{R}$ is increasing, $f(0) \geq 0$, and one of the following:

$\left(f_{2}\right)|\partial f(u) / \partial u| \leq C_{1}|u|^{\beta_{1}}+C_{2}|u|^{\beta_{2}}$, where $u^{\beta_{1}}, u^{\beta_{2}} \in L^{n /(2 m)}\left(\mathbb{R}_{+}^{n}\right), \beta_{1}$ is some nonnegative constant, $\beta_{2}$ is some nonpositive constant and $C_{1}, C_{2}$ are nonnegative constants, or

$\left(\tilde{f}_{2}\right) f^{\prime}(\cdot)$ is nondecreasing and $f^{\prime}(u) \in L^{n /(2 m)}\left(\mathbb{R}_{+}^{n}\right)$.

Obviously, we should assume that $f \not \equiv 0$.

We prove the following theorem by the method of moving planes in integral form.

Theorem 1. If $\left(f_{1}\right)$ and either $\left(f_{2}\right)$ or $\left(\tilde{f}_{2}\right)$ hold, and the function $u \in L^{r}\left(\mathbb{R}_{+}^{n}\right)$ for some $r>n /(n-2 m)$ is a nonnegative solution of (1), then $u$ is trivial.

The integral equation (1) is closely related to the higher order elliptic equations

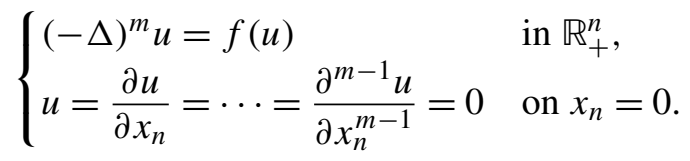

The weak solutions of (2) in the Sobolev space $H_{0}^{m}\left(\mathbb{R}_{+}^{n}\right)$ satisfy

$$
\langle u, v\rangle_{m}=\int_{\mathbb{R}_{+}^{n}} f(u(x)) v(x) d x \quad \text { for all } v \in H_{0}^{m}\left(\mathbb{R}_{+}^{n}\right),
$$

where

$$
\langle u, v\rangle_{m}= \begin{cases}\int_{\mathbb{R}_{+}^{n}} \Delta^{m / 2} u \Delta^{m / 2} v d x & m \text { even, } \\ \int_{\mathbb{R}_{+}^{n}} \nabla \Delta^{(m-1) / 2} u \nabla \Delta^{(m-1) / 2} v d x & m \text { odd. }\end{cases}
$$

Under the conditions that $u \in C^{2 m-1}\left(\overline{\mathbb{R}}_{+}^{n}\right) \cap W_{\text {loc }}^{2 m, p}\left(\mathbb{R}_{+}^{n}\right)$ for some $p>(n) / 2 m$ and $u$ and all partial derivatives of $u$ of order less than or equal to $2 m-1$ are bounded, Reichel and Weth [2009] prove that (2) implies (1). Therefore, Theorem 1 implies the following:

Corollary 1. Suppose $\left(f_{1}\right)$ and either $\left(f_{2}\right)$ or $\left(\tilde{f}_{2}\right)$ hold, $p>n /(2 m), u \in L^{r}\left(\mathbb{R}_{+}^{n}\right)$ for some $r>n /(n-2 m)$ and $u \in C^{2 m-1}\left(\overline{\mathbb{R}}_{+}^{n}\right) \cap W_{\text {loc }}^{2 m, p}\left(\mathbb{R}_{+}^{n}\right)$ is a nonnegative solution of (2). Moreover, suppose that $u$ and all the partial derivatives of $u$ of order less than or equal to $2 m-1$ are bounded. Then $u$ is trivial.

We also consider the properties of nonnegative solutions of the following systems of integral equations:

$$
\left.u_{i}(x)=\int_{\mathbb{R}_{+}^{n}} G(x, y) f_{i}\left(y^{\prime}, y_{n}, u_{1}, \ldots, u_{N}\right)\right) d y, \quad i=1, \ldots, N,
$$


where $y^{\prime}=\left(y_{1}, \ldots, y_{n-1}\right)$. Assume $f_{i}\left(x^{\prime}, x_{n}, u_{1}, \ldots, u_{N}\right)(i=1, \ldots, N)$ is nondecreasing with respect to every $u_{j}(j=1, \ldots, N)$, nonincreasing with respect to $\left|x^{\prime}\right|$ and nondecreasing with respect to $x_{n}$. Moreover, assume that $f_{i}$ satisfies

$$
\left(f_{i}^{1}\right) f_{i}:[0, \infty) \rightarrow \mathbb{R}, f_{i}\left(x^{\prime}, x_{n}, 0\right) \geq 0,
$$

and either of the following:

$\left(f_{i}^{2}\right)\left|\partial f_{i} / \partial u_{j}\right| \leq C_{i j}^{0}\|u\|^{\beta_{i j}^{0}}+C_{i j}^{1}\|u\|^{\beta_{i j}^{1}}+g_{i j}(x)$, or

$\left(\tilde{f}_{i}^{2}\right) \partial f_{i} / \partial u_{j}$ is nondecreasing with respect to $u_{j}$ and $\partial f_{i} / \partial u_{j} \in L^{n /(2 m)}\left(\mathbb{R}_{+}^{n}\right)$.

In these conditions, $\|u\|:=\sqrt{u_{1}^{2}+\cdots+u_{N}^{2}}$ and we have assumed that

$$
\|u\|^{\beta_{i j}^{0}},\|u\|^{\beta_{i j}^{1}}, g_{i j} \in L^{n /(2 m)}\left(\mathbb{R}_{+}^{n}\right),
$$

$C_{i j}^{0}$ and $C_{i j}^{1}$ are nonnegative constants, $\beta_{i j}^{0}$ is a nonnegative constant and $\beta_{i j}^{1}$ is a nonpositive constant. Obviously, we also assume that $f_{i} \not \equiv 0$ for $i=1, \ldots, N$.

Definition (see [Jin and Li 2006]). Functions $f_{1}, \ldots, f_{N}$ are essentially related if

$$
\sum_{l=1}^{l_{0}} f_{i_{l}}\left(x^{\prime}, x_{n}, u_{1}, u_{2}, \ldots, u_{N}\right) \neq \sum_{l=1}^{l_{0}} f_{i_{l}}\left(x^{\prime}, x_{n}, v_{1}, v_{2}, \ldots, v_{N}\right),
$$

provided that $u_{i} \leq v_{i}$ for $i=1, \ldots, N$ and $u_{j}<v_{j}$ for $j \in S$, where $S=\{1, \ldots, N\} \backslash$ $\left\{i_{1}, \ldots, i_{l_{0}}\right\}$.

With this definition, the systems cannot be divided into independent subsystems.

We prove the following theorem with the moving plane method in integral form.

Theorem 2. If $\left(f_{i}^{1}\right)$ and either $\left(f_{i}^{2}\right)$ or $\left(\tilde{f}_{i}^{2}\right)$ hold and the function $u_{i} \in L^{r}\left(\mathbb{R}_{+}^{n}\right)$ for some $r>n /(n-2 m)$ is a nonnegative solution of (4), then $u_{i}$ is trivial for $i=1, \ldots, N$.

Similarly, the system (4) of integral equations is closely related to following system of higher order elliptic equations:

$$
\begin{cases}(-\Delta)^{m} u_{i}=f_{i}\left(x^{\prime}, x_{n}, u_{1}, \ldots, u_{N}\right) & \text { in } \mathbb{R}_{+}^{n}, i=1, \cdots, N, \\ u_{i}=\frac{\partial u_{i}}{\partial x_{n}}=\cdots=\frac{\partial^{m-1} u_{i}}{\partial x_{n}^{m-1}}=0 & \text { on } x_{n}=0 .\end{cases}
$$

It is well known that the moving plane method was first developed by the Soviet mathematician Alexandrov in the 1950s. It was further developed by Serrin [1971], Gidas, Ni and Nirenberg [1979], Caffarelli, Gidas and Spruck [1989], Chen and Li [1991], Chang and Yang [1997], Wei and Xu [1999] and many others. Recently, Chen, Li and Ou [2005; 2006] applied the moving plane method to integral equations to obtain the symmetry, monotonicity and nonexistence properties of the solutions to the integral equations. Instead of extensive use of the maximum principle of differential equations, the moving plane method in integral form explores 
various specific features of the integral equation itself. (See also the work of $\mathrm{Li}$ [2004] on the moving sphere method in integral form.) Subsequently, more work has been done in the direction of the moving plane method in integral form: see [Jin and Li 2006; Ma and Chen 2006; 2008; Qing and Raske 2006; Hang 2007; Li and Ma 2008] and others. Nevertheless, all work on the moving plane method in integral form has been on the whole space $\mathbb{R}^{n}$ (or on a ball in [Chen and Zhu 2011]). In this paper, we will adapt the moving plane method in integral form in a half-space to prove the axial symmetry of nonnegative solutions to a class of integral equations associated to the Dirichlet problem of polyharmonic equations on a half-space.

By virtue of the Hardy-Littlewood-Sobolev inequality or its general form, the weighted Hardy-Littlewood-Sobolev inequality, and comparison of the solution and its reflection with the plane, we can start moving the plane from infinity. Furthermore the plane must be moved to a critical point. As a result, symmetry and monotonicity properties are derived.

We also obtain regularity results for

$$
u(x)=\int_{\mathbb{R}_{+}^{n}} G(x, y)|u|^{p-1} u d y
$$

in the case of $n>2 m$ and $p>n /(n-2 m)$. The method we use here is called "regularity lifting" based on the contraction mapping theorem. It is an elegant and powerful tool in obtaining regularity of solutions. (See [Chen and Li 2010], and also Section 3 below for more details.)

Theorem 3. Let $u(x)$ be a solution of (6). Assume that $p>n /(n-2 m)$ and $u(x) \in L^{(p-1) n /(2 m)}\left(\mathbb{R}_{+}^{n}\right)$. Then $u$ is bounded in $\mathbb{R}_{+}^{n}$ and moreover in $L^{s}\left(\mathbb{R}_{+}^{n}\right)$ for $s>n /(n-2 m)$.

Next, we consider the nonnegative solutions in (6), that is,

$$
u(x)=\int_{\mathbb{R}_{+}^{n}} G(x, y) u^{p}(y) d y .
$$

Corollary 2. Assume $p>n /(n-2 m)$ and let $u(x)$ be the nonnegative solution in $(7)$ and $u(x) \in L^{(p-1) n /(2 m)}\left(\mathbb{R}_{+}^{n}\right)$. Then $u$ is trivial.

We further study the properties of solutions in (7) under the weaker assumption that $u \in L_{\text {loc }}^{2 n /(n-2 m)}\left(\mathbb{R}_{+}^{n}\right)$. We obtain that

Theorem 4. Let $u(x)$ be the nonnegative solution in (7) with $u \in L_{\mathrm{loc}}^{2 n /(n-2 m)}\left(\mathbb{R}_{+}^{n}\right)$ and assume $1<p<(n+2 m) /(n-2 m)$. Then $u(x)$ only depends on $x_{n}$.

The paper is arranged as follows. In Section 2, we present some properties of Green's function for polyharmonic operators in a half-space. Section 3 is devoted to the proof of Theorem 1 using the method of moving planes in integral form. 
In Section 4, we verify Theorem 2 with a similar technique of moving planes in integral form. We establish Theorem 3 by the contraction mapping method in Section 5. Theorem 4 is obtained in Section 6. In this paper $C$ denotes a positive constant, which may vary from line to line.

\section{Properties of Green's function}

In this section, we introduce some results about the Green's function $G=G(x, y)$ of $(-\Delta)^{m}$ in $\mathbb{R}_{+}^{n}$ corresponding to a Dirichlet boundary condition. For fixed $y \in \mathbb{R}_{+}^{N}$,

$$
\begin{cases}(-\Delta)^{m} G(x, y)=\delta(x-y) & \text { in } \mathbb{R}_{+}^{n}, \\ G=\frac{\partial G}{\partial x_{n}}=\cdots=\frac{\partial^{m-1} G}{\partial x_{n}^{m-1}}=0 & \text { on } x_{n}=0 .\end{cases}
$$

Define

$$
\begin{aligned}
& d(x, y)=|x-y|^{2} \quad \text { for } x, y \in \mathbb{R}_{+}^{n}, \\
& \theta(x, y)= \begin{cases}x_{n} y_{n} & \text { if } x, y \in \mathbb{R}_{+}^{n}, \\
0 & x \notin \mathbb{R}_{+}^{n} \text { or } y \notin \mathbb{R}_{+}^{n} .\end{cases}
\end{aligned}
$$

Using a rescaling argument from the Green's function of a polyharmonic elliptic equation in the ball [Boggio 1905] (see also [Bachar et al. 2004]), $G=G(x, y)$ has the form

$$
\begin{aligned}
G(x, y) & =K_{n}^{m}|x-y|^{2 m-n} \int_{0}^{4 \theta(x, y) /|x-y|^{2}} \frac{z^{m-1}}{(z+1)^{n / 2}} d z \\
& =K_{n}^{m} H(d(x, y), \theta(x, y)) .
\end{aligned}
$$

Here $K_{n}^{m}$ is a positive constant and

$$
H:(0, \infty) \times[0, \infty) \rightarrow \mathbb{R}, \quad H(s, t)=s^{m-n / 2} \int_{0}^{4 t / s} \frac{z^{m-1}}{(z+1)^{n / 2}} d z
$$

with

$$
d(x, y)=s, \quad \theta(x, y)=t .
$$

We now introduce some notation which will be used extensively in this paper. Let $x=\left\{x_{1}, \ldots, x_{i}, \ldots, x_{n}\right\}$ for $1 \leq i \leq n$, let $T_{\lambda}^{i}=\left\{x \mid x_{i}=\lambda\right\}$ and let $x_{\lambda}^{i}=$ $\left\{x_{1}, \ldots, 2 \lambda-x_{i}, \ldots, x_{n}\right\}$ be the reflection of the point $x$ about the plane $T_{\lambda}^{i}$. Set $\Sigma_{\lambda}^{i}=\left\{x \in \mathbb{R}_{+}^{n} \mid x_{i}<\lambda\right\}$. If $1 \leq i<n$ then $\lambda$ can be any real number. For $i=n$, since we will move the plane from $x_{n}=0$ to positive infinity, we only consider the case that $\lambda$ is positive. In this case, we introduce $\left(\Sigma_{\lambda}^{n}\right)^{C}=\left\{x_{\lambda} \mid x \in \Sigma_{\lambda}^{n}\right\}$.

To simplify the presentation, we will drop the superscript $i$ from $T_{\lambda}^{i}, \Sigma_{\lambda}^{i}, x_{\lambda}^{i}$, etc. when $1 \leq i<n$ or $i=n$ is given and no confusion is caused. 
We will prove the following properties for $G(x, y)$ in a half-space which will be used in next section. See [Berchio et al. 2008; Chen and Zhu 2011] for a similar lemma on Green's function on a ball.

Lemma 1. (i) Let $\lambda \in(-\infty, 0)$. For any $x, y \in \Sigma_{\lambda}, x \neq y$,

$$
\begin{aligned}
G\left(x_{\lambda}, y_{\lambda}\right) & >\max \left\{G\left(x_{\lambda}, y\right), G\left(x, y_{\lambda}\right)\right\}, \\
G\left(x_{\lambda}, y_{\lambda}\right)-G(x, y) & =G\left(x_{\lambda}, y\right)-G\left(x, y_{\lambda}\right)=0 \quad \text { if } 1 \leq i<n .
\end{aligned}
$$

(ii) Let $\lambda \in(0, \infty)$. For any $x, y \in \Sigma_{\lambda}, x \neq y$,

$$
G\left(x_{\lambda}, y_{\lambda}\right)-G(x, y)>\left|G\left(x_{\lambda}, y\right)-G\left(x, y_{\lambda}\right)\right| \quad \text { if } i=n \text {. }
$$

(iii) Let $\lambda \in(0, \infty)$. For any $x \in \Sigma_{\lambda}, y \in \mathbb{R}_{+}^{n} \backslash\left(\Sigma_{\lambda} \cup \Sigma_{\lambda}^{C}\right)$,

$$
G(x, y)<G\left(x_{\lambda}, y\right) \quad \text { if } i=n .
$$

Proof. (i) For $x, y \in \Sigma_{\lambda}$, obviously $d\left(x_{\lambda}, y_{\lambda}\right)<d\left(x, y_{\lambda}\right)$. Since $\theta$ is only dependent on the $n$-th variable, in the case $1 \leq i<n$,

$$
\theta\left(x_{\lambda}, y_{\lambda}\right)=\theta\left(x, y_{\lambda}\right)=\theta\left(x_{\lambda}, y\right)=\theta(x, y) .
$$

In the case $i=n$,

$$
\theta\left(x_{\lambda}, y_{\lambda}\right)>\max \left(\theta\left(x, y_{\lambda}\right), \theta\left(x_{\lambda}, y\right)\right) \geq \min \left(\theta\left(x, y_{\lambda}\right), \theta\left(x_{\lambda}, y\right)\right)>\theta(x, y) .
$$

We compute

For $s, t>0$,

$$
\begin{aligned}
H(s, t) & =s^{m-n / 2} \int_{0}^{4 t / s} \frac{z^{m-1}}{(z+1)^{n / 2}} d z \\
& =\int_{0}^{4 t} \frac{z^{m-1}}{(z+s)^{n / 2}} d z .
\end{aligned}
$$

$$
\begin{aligned}
\frac{\partial H}{\partial s} & =-\frac{n}{2} \int_{0}^{4 t} \frac{z^{m-1}}{(z+s)^{n / 2+1}} d z<0, \\
\frac{\partial H}{\partial t} & =\frac{4(4 t)^{m-1}}{(4 t+s)^{n / 2}}>0, \\
\frac{\partial^{2} H}{\partial t \partial s} & =\frac{-2 n(4 t)^{m-1}}{(t+s)^{n / 2+1}}<0 .
\end{aligned}
$$

From (14), (15), (16) and (17), we arrive at (10).

In the case $1 \leq i<n$, since $d\left(x_{\lambda}, y_{\lambda}\right)=d(x, y)$ and $d\left(x, y_{\lambda}\right)=d\left(x_{\lambda}, y\right)$, and moreover, $\theta(x, y)$ is a function in $x_{n}$ and $y_{n}$, it is easy to verify (11). 
(ii) If $i=n$, from (15), (18),

$$
\begin{aligned}
G\left(x_{\lambda}, y_{\lambda}\right)-G(x, y) & =K_{n}^{m} \int_{\theta(x, y)}^{\theta\left(x_{\lambda}, y_{\lambda}\right)} \frac{\partial H(d(x, y), t)}{\partial t} d t \\
& >K_{n}^{m} \int_{\theta(x, y)}^{\theta\left(x_{\lambda}, y_{\lambda}\right)} \frac{\partial H\left(d\left(x_{\lambda}, y\right), t\right)}{\partial t} d t \\
& \geq K_{n}^{m} \int_{\min \left(\theta\left(x, y_{\lambda}\right), \theta\left(x_{\lambda}, y\right)\right)}^{\max \left(\theta\left(x, y_{\lambda}\right), \theta\left(x_{\lambda}, y\right)\right)} \frac{\partial H\left(d\left(x_{\lambda}, y\right), t\right)}{\partial t} d t \\
& =K_{n}^{m}\left|H\left(d\left(x_{\lambda}, y\right), \theta\left(x_{\lambda}, y\right)\right)-H\left(d\left(x, y_{\lambda}\right), \theta\left(x, y_{\lambda}\right)\right)\right| \\
& =\left|G\left(x_{\lambda}, y\right)-G\left(x, y_{\lambda}\right)\right|,
\end{aligned}
$$

which confirms (12).

(iii) For $i=n$, if $x \in \Sigma_{\lambda}$ and $y \in \mathbb{R}_{+}^{n} \backslash\left(\Sigma_{\lambda} \cup \Sigma_{\lambda}^{C}\right)$, we have

$$
d(x, y)>d\left(x_{\lambda}, y\right)
$$

and

$$
\theta(x, y)<\theta\left(x_{\lambda}, y\right) .
$$

Then (13) follows immediately from (16) and (17).

\section{Proof of Theorem 1}

Let $u^{\lambda}(x)=u\left(x_{\lambda}\right)$. Once again, we have dropped the superscript $i$ from $x_{\lambda}^{i}$, etc...

Lemma 2. The following equality holds:

$$
u(x)-u\left(x_{\lambda}\right) \leq \int_{\Sigma_{\lambda}}\left(G\left(x_{\lambda}, y_{\lambda}\right)-G\left(x, y_{\lambda}\right)\right)\left(f(u(y))-f\left(u^{\lambda}(y)\right)\right) .
$$

Proof. First consider the case $1 \leq i<n$. In this situation,

$$
\begin{gathered}
u(x)=\int_{\Sigma_{\lambda}} G(x, y) f(u(y)) d y+\int_{\Sigma_{\lambda}} G\left(x, y_{\lambda}\right) f\left(u^{\lambda}(y)\right) d y, \\
u^{\lambda}(x)=\int_{\Sigma_{\lambda}} G\left(x_{\lambda}, y\right) f(u(y)) d y+\int_{\Sigma_{\lambda}} G\left(x_{\lambda}, y_{\lambda}\right) f\left(u^{\lambda}(y)\right) d y .
\end{gathered}
$$

Combining this with (11) in Lemma 1, we derive

$$
u(x)-u\left(x_{\lambda}\right)=\int_{\Sigma_{\lambda}}\left(G\left(x_{\lambda}, y_{\lambda}\right)-G\left(x, y_{\lambda}\right)\right)\left(f(u(y))-f\left(u^{\lambda}(y)\right)\right) d y .
$$


Now assume $i=n$.

$$
\begin{aligned}
u(x)=\int_{\Sigma_{\lambda}} G(x, y) f(u(y)) d y+\int_{\Sigma_{\lambda}} G\left(x, y_{\lambda}\right) f\left(u^{\lambda}(y)\right) d y & \\
& +\int_{\mathbb{R}_{+}^{n} \backslash\left(\Sigma_{\lambda} \cup \Sigma_{\lambda}^{C}\right)} G(x, y) f(u(y)) d y, \\
u^{\lambda}(x)=\int_{\Sigma_{\lambda}} G\left(x_{\lambda}, y\right) f(u(y)) d y+\int_{\Sigma_{\lambda}} G\left(x_{\lambda}, y_{\lambda}\right) f\left(u^{\lambda}(y)\right) d y & +\int_{\mathbb{R}_{+}^{n} \backslash\left(\Sigma_{\lambda} \cup \Sigma_{\lambda}^{C}\right)} G\left(x_{\lambda}, y\right) f(u(y)) d y .
\end{aligned}
$$

From (12), (13) in Lemma 1 and the property of $f_{i}$, we derive

$$
u(x)-u\left(x_{\lambda}\right) \leq \int_{\Sigma_{\lambda}}\left(G\left(x_{\lambda}, y_{\lambda}\right)-G\left(x, y_{\lambda}\right)\right)\left(f(u(y))-f\left(u^{\lambda}(y)\right)\right) d y .
$$

This completes the proof of the lemma.

Lemma 3 (equivalent form of the Hardy-Littlewood-Sobolev inequality). Assume $0<\alpha<n$ and $\Omega \subset \mathbb{R}^{n}$. Let $g \in L^{n p /(n+\alpha p)}(\Omega)$ for $n /(n-\alpha)<p<\infty$. Define

$$
\operatorname{Tg}(x)=\int_{\Omega} \frac{1}{|x-y|^{n-\alpha}} g(y) d y \text {. }
$$

Then

$$
\|T g\|_{L^{p}(\Omega)} \leq C(n, p, \alpha)\|g\|_{L^{n p /(n+\alpha p)}(\Omega)} .
$$

The proof of this lemma is standard and follows from the $L^{n p /(n+\alpha p)} \rightarrow L^{p}$ boundedness for the fractional integral operator of order $\alpha$ when $p>n /(n-\alpha)$ (see, for example, [Stein 1970]).

Next we will prove $u(x)$ is axially symmetric. In the proof of nonexistence of solutions, we only need to show that $u(x)$ is increasing in the $x_{n}$ direction. For the sake of completeness, we present the whole picture in half-space of the moving plane method in integral form. This way of showing axial symmetry is also is used in proof of Theorem 4 . We first show that $u(x)$ is radially symmetric with respect to some $x_{0} \in \mathbb{R}^{n-1}$ for any fixed $x_{n}$. Then we prove that $u(x)$ is nondecreasing in the $x_{n}$ direction. To prove radial symmetry, there are two steps in carrying out the process of moving planes. In Step 1, we need to show that the plane can be moved near infinity, that is, we will show that $u(x) \leq u\left(x_{\lambda}\right)$ for sufficiently negative $\lambda$. In Step 2, we prove that the plane has to move to a critical point. By a contradiction argument, radial symmetry is obtained. To prove $u(x)$ is nondecreasing, we only need to carry out Step 1 in the $x_{n}$ direction.

Lemma 4. If $\left(f_{1}\right)$ and either $\left(f_{2}\right)$ or $\left(\tilde{f}_{2}\right)$ hold, $u \in L^{r}\left(\mathbb{R}_{+}^{n}\right)$ for some $r>n /(n-2 m)$ is a nonnegative solution of (1), then $u$ is axially symmetric with respect to some line parallel to the $x_{n}$-axis and $u(x)$ is nondecreasing in the $x_{n}$ direction. 
Proof. First consider the case $1 \leq i<n$. Without loss of generality, let $i=1$. Start to move the plane in the $x_{1}$ coordinate. Proving the symmetry of solutions in the $x_{1}$ coordinate and taking the same steps with all coordinates except $x_{n}$ gives radial symmetry of solutions with respect to some $x_{0} \in R^{n-1}$ with fixed $x_{n}$.

Step 1: Define

$$
\begin{aligned}
\Sigma_{\lambda}^{-} & =\left\{x \in \Sigma_{\lambda} \mid u(x)>u^{\lambda}(x)\right\}, \\
w^{\lambda}(x) & =u(x)-u^{\lambda}(x) .
\end{aligned}
$$

By (10) in Lemma 1, positivity of the Green's function, the properties of $f$ and Lemma 2,

$$
\begin{aligned}
u(x)-u\left(x_{\lambda}\right) \leq & \int_{\Sigma_{\lambda} \backslash \Sigma_{\lambda}^{-}}\left(G\left(x_{\lambda}, y_{\lambda}\right)-G\left(x, y_{\lambda}\right)\right)\left(f(u(y))-f\left(u^{\lambda}(y)\right)\right) d y \\
& \quad+\int_{\Sigma_{\lambda}^{-}}\left(G\left(x_{\lambda}, y_{\lambda}\right)-G\left(x, y_{\lambda}\right)\right)\left(f(u(y))-f\left(u^{\lambda}(y)\right)\right) d y \\
\leq & \int_{\Sigma_{\lambda}^{-}} G\left(x_{\lambda}, y_{\lambda}\right)\left(f(u(y))-f\left(u^{\lambda}(y)\right)\right) d y \\
\leq & C \int_{\Sigma_{\lambda}^{-}}|x-y|^{2 m-n} \int_{0}^{4 x_{n} y_{n} /|x-y|^{2}} \frac{z^{m-1}}{(z+1)^{n / 2}} d z\left(f(u(y))-f\left(u^{\lambda}(y)\right)\right) d y \\
\leq & C \int_{\Sigma_{\lambda}^{-}}|x-y|^{2 m-n}\left(f(u(y))-f\left(u^{\lambda}(y)\right)\right) d y .
\end{aligned}
$$

By the Hardy-Littlewood-Sobolev inequality, the mean value theorem, and the assumption that $u \in L^{r}\left(\mathbb{R}_{+}^{n}\right)$ for some $r>n /(n-2 m)$, we deduce

$$
\left\|w^{\lambda}\right\|_{L^{r}\left(\Sigma_{\lambda}^{-}\right)} \leq C\left\|\frac{\partial f\left(\theta u+(1-\theta) u^{\lambda}\right)}{\partial u} w^{\lambda}\right\|_{L^{n r /(n+2 m r)}\left(\Sigma_{\lambda}^{-}\right)},
$$

where $0<\theta<1$. Furthermore, by Hölder's inequality, we get

$$
\left\|w^{\lambda}\right\|_{L^{r}\left(\Sigma_{\lambda}^{-}\right)} \leq C\left\|\frac{\partial f\left(\theta u+(1-\theta) u^{\lambda}\right)}{\partial u}\right\|_{L^{n /(2 m)}\left(\Sigma_{\lambda}^{-}\right)}\left\|w^{\lambda}\right\|_{L^{r}\left(\Sigma_{\lambda}^{-}\right)} .
$$

From property $\left(f_{2}\right)$ of the function $f$, if $\lambda$ is sufficiently negative,

$$
C\left\|\frac{\partial f\left(\theta u+(1-\theta) u^{\lambda}\right)}{\partial u}\right\|_{L^{n /(2 m)}\left(\Sigma_{\lambda}^{-}\right)} \leq \frac{1}{2},
$$

then

$$
\left\|w^{\lambda}\right\|_{L^{r}\left(\Sigma_{\lambda}^{-}\right)} \leq \frac{1}{2}\left\|w^{\lambda}\right\|_{L^{r}\left(\Sigma_{\lambda}^{-}\right)},
$$


which implies that $\left\|w^{\lambda}\right\|_{L^{r}\left(\Sigma_{\lambda}^{-}\right)}=0$. Therefore, $\Sigma_{\lambda}^{-}$must be of measure zero. Thus, $u(x) \leq u^{\lambda}(x)$ a.e. in $\Sigma_{\lambda}$ for sufficiently negative $\lambda$.

Step 2: Continue to move the plane $x_{1}=\lambda$ to the right as long as Step 1 holds. We claim that there exists some critical point

$$
\lambda_{0}=\sup _{\lambda}\left\{u^{\lambda}(x) \geq u(x) \mid-\infty<\lambda<0, x \in \Sigma_{\lambda}\right\}
$$

such that $w^{\lambda_{0}} \equiv 0$.

If $\lambda_{0}=0$ in Step 1 , then $u(x) \leq u\left(x_{\lambda_{0}}\right)$ in $\Sigma_{\lambda_{0}}$. Move the plane from positive infinity to the origin and argue in the same way as in Step 1 . If $\lambda_{0}=0$ again, it is obvious that $u(x) \equiv u\left(x_{\lambda_{0}}\right)$. Hence, $w^{\lambda_{0}}(x) \equiv 0$.

If $\lambda_{0}<0$, we claim that

$$
w^{\lambda_{0}}(x)<0 \quad \text { or } \quad w^{\lambda_{0}}(x) \equiv 0 \quad \text { for } x \in \Sigma_{\lambda_{0}} .
$$

Suppose that there exists some $x_{0}$ in $\Sigma_{\lambda_{0}}$ such that $w^{\lambda_{0}}\left(x_{0}\right)=0$, but $w^{\lambda_{0}} \not \equiv 0$. By (11) in Lemma 1,

$$
u\left(x_{0}\right)-u_{\lambda_{0}}\left(x_{0}\right)=\int_{\Sigma_{\lambda_{0}}}\left(G\left(x_{\lambda_{0}}, y_{\lambda_{0}}\right)-G\left(x, y_{\lambda_{0}}\right)\right)\left(f(u(y))-f\left(u_{\lambda_{0}}(y)\right)\right) d y .
$$

Moreover, by (10), $f(u(y)) \equiv f\left(u\left(y_{\lambda_{0}}\right)\right.$. This contradicts with the fact that the function $f$ satisfies $\left(f_{1}\right)$, which verifies the claim.

Next, we show the plane can be moved to the right a little bit farther if $w^{\lambda_{0}}(x)<0$. By the assumption $\left(f_{2}\right)$ on the function $f$, for any small $\epsilon$, there exists a large enough ball $\mathbb{B}_{R}(0)$ such that

$$
\left\|\frac{\partial f\left(\theta u+(1-\theta) u_{\lambda}\right)}{\partial u}\right\|_{L^{n /(2 m)}\left(\mathbb{R}_{+}^{n} \backslash \mathbb{B}_{R}\right)}<\epsilon .
$$

From Lusin's theorem, for any $\delta$, there exists a closed set $F_{\delta}$ such that $\left.w_{\lambda_{0}}\right|_{F_{\delta}}$ is continuous, with $F_{\delta} \subset E:=\mathbb{B}_{R}(0) \cap \Sigma_{\lambda_{0}}$ and $m\left(E-F_{\delta}\right)<\delta$. As $w^{\lambda_{0}}(x)<0$ in the interior of $\Sigma_{\lambda_{0}}, w^{\lambda_{0}}(x)<0$ in $F_{\delta}$.

Choosing $\epsilon_{1}$ sufficiently small, for any $\lambda \in\left[\lambda_{0}, \lambda_{0}+\epsilon_{1}\right)$, we have

$$
w^{\lambda}(x)<0 \text { for all } x \in F_{\delta}
$$

by continuity. It follows that for such $\lambda$,

$$
\Sigma_{\lambda}^{-} \subset M:=\left(\mathbb{R}_{+}^{n} \backslash \mathbb{B}_{R}(0)\right) \cup\left(E \backslash F_{\delta}\right) \cup\left(\left(\Sigma_{\lambda} \backslash \Sigma_{\lambda_{0}}^{-}\right) \cap \mathbb{B}_{R}(0)\right) .
$$

Choosing $\epsilon, \delta$ and $\epsilon_{1}$ small enough and using absolute continuity of integration, we derive

$$
C\left\|\frac{\partial f\left(u_{\lambda}+\theta w^{\lambda}\right)}{\partial u}\right\|_{L^{n /(2 m)}(M)} \leq \frac{1}{2} .
$$


Consequently from (20), $\left\|w^{\lambda}(x)\right\|_{L^{q}\left(\Sigma_{\lambda}^{-}\right)}=0$. Then, $\Sigma_{\lambda}^{-}$must be of measure zero, which contradicts the definition of $\lambda_{0}$. Hence, $w^{\lambda_{0}} \equiv 0$.

This completes Steps 1 and 2 for $1 \leq i<n$.

For the case $i=n$, start moving the plane from $x_{n}=0$ as in the case $1 \leq i<n$. Choosing $\lambda>0$ sufficiently small, Step 1 is carried out similarly, which implies that $w^{\lambda}(x) \leq 0$. Next we prove that if $w^{\lambda}(x) \not \equiv 0$,

$$
\lambda_{0}=\sup _{\lambda}\left\{u^{\lambda}(x)>u(x), \lambda>0, x \in \Sigma_{\lambda}\right\}=\infty .
$$

If not, then $\lambda_{0}<\infty$. It is known that $w^{\lambda_{0}}(x)<0$ or $w^{\lambda_{0}}(x) \equiv 0$ for any $x \in \Sigma_{\lambda_{0}}$. Hence, $w^{\lambda}(x)<0$ for any $\lambda$. If $w^{\lambda}(x) \equiv 0$, since $u\left(x^{\prime}, x_{n}\right)=0$ on $x_{n}=0$, then $u(x) \equiv 0$. Therefore $u(x)$ is nondecreasing in the $x_{n}$ direction. Thus we have completed the proof of Lemma 4.

Proof of Theorem 1. Since $u(x)$ is nondecreasing in the $x_{n}$ direction by Lemma 4 and $u \in L^{r}\left(\mathbb{R}_{+}^{n}\right)$ for some $r>n /(n-2 m)$ by assumption, then for any $a \in \mathbb{R}_{+}$,

$$
\int_{\mathbb{R}_{+}^{n}}\left|u\left(x^{\prime}, x_{n}\right)\right|^{r} d x^{\prime} d x_{n} \geq \int_{\mathbb{R}^{n-1}} \int_{a}^{\infty}\left|u\left(x^{\prime}, a\right)\right|^{r} d x^{\prime} d x_{n} .
$$

The integrability of nonnegative $u$ implies $u\left(x^{\prime}, a\right)=0$ for any $a$ and $x^{\prime} \in \mathbb{R}^{n-1}$. Hence $u(x)=0$ in $\mathbb{R}_{+}^{n}$.

\section{Proof of Theorem 2}

Lemma 5. If $\left(f_{i}^{1}\right)$ and either $\left(f_{i}^{2}\right)$ or $\left(\tilde{f}_{i}^{2}\right)$ hold and $u_{i} \in L^{r}\left(\mathbb{R}_{+}^{n}\right)$ for some $r>$ $n /(n-2 m)$ is the nonnegative solution of $(4)$, then $u_{i}$ is axially symmetric with respect to some line parallel to the $x_{n}$-axis and $u_{i}(x)$ is nondecreasing in the $x_{n}$ direction for $i=1, \ldots, N$.

Proof. Step 1: We proceed with the same process as in the proof of Theorem 1. First consider all coordinates except $x_{n}$. Without loss of generality, move the plane in the $x_{1}$ coordinate. Let

$$
\begin{aligned}
\Sigma_{\lambda}^{j} & =\left\{x \in \Sigma_{\lambda} \mid u_{j}(x)>u_{j}\left(x_{\lambda}\right)\right\}, \\
w_{i}^{\lambda}(x) & =u_{i}(x)-u_{i}\left(x_{\lambda}\right) .
\end{aligned}
$$

The same technique as in Lemma 2 and properties $\left(f_{i}^{1}\right)$ of the functions $f_{i}$ give

$$
\begin{aligned}
& w_{i}^{\lambda}(x) \\
& \quad \leq \int_{\Sigma_{\lambda}}\left(G\left(x_{\lambda}, y_{\lambda}\right)-G\left(x, y_{\lambda}\right)\right)\left(f_{i}\left(y^{\prime}, y_{n}, u_{1}, \ldots, u_{N}\right)-f_{i}\left(y^{\prime}, y_{n}, u_{1}^{\lambda}, \ldots, u_{N}^{\lambda}\right)\right) \\
& \quad=\sum_{j=1}^{N} \int_{\Sigma_{\lambda}}\left(G\left(x_{\lambda}, y_{\lambda}\right)-G\left(x, y_{\lambda}\right)\right) K_{i, j}(y, \lambda) d y
\end{aligned}
$$




$$
\begin{aligned}
& =\sum_{j=1}^{N} \int_{\Sigma_{\lambda} \backslash \Sigma_{\lambda}^{j}}\left(G\left(x_{\lambda}, y_{\lambda}\right)-G\left(x, y_{\lambda}\right)\right) K_{i, j}(y, \lambda) d y \\
& \quad+\sum_{j=1}^{N} \int_{\Sigma_{\lambda}^{j}}\left(G\left(x_{\lambda}, y_{\lambda}\right)-G\left(x, y_{\lambda}\right)\right) K_{i, j}(y, \lambda) d y \\
& \leq \sum_{j=1}^{N} \int_{\Sigma_{\lambda}^{j}} G\left(x_{\lambda}, y_{\lambda}\right) K_{i, j}(y, \lambda) d y,
\end{aligned}
$$

where

$$
\begin{aligned}
& K_{i, j}(y, \lambda) \\
& \quad=f_{i}\left(y^{\prime}, y_{n}, u_{1}^{\lambda}, \ldots, u_{j-1}^{\lambda}, u_{j}, \ldots, u_{N}\right)-f_{i}\left(y^{\prime}, y_{n}, u_{1}^{\lambda}, \ldots, u_{j-1}^{\lambda}, u_{j}^{\lambda}, \ldots, u_{N}\right) .
\end{aligned}
$$

Estimating in the same way as in the proof of Lemma 4,

$$
u_{i}(x)-u_{i}\left(x_{\lambda}\right) \leq C \sum_{j=1}^{N} \int_{\Sigma_{\lambda}^{j}}|x-y|^{2 m-n} K_{i, j}(y, \lambda) d y .
$$

By the Hardy-Littlewood-Sobolev inequality, the mean value theorem and the assumption that $u \in L^{r}\left(\mathbb{R}_{+}^{n}\right)$ for some $r>n /(n-2 m)$, we deduce

$$
\left\|w_{i}^{\lambda}\right\|_{L^{r}\left(\Sigma_{\lambda}^{i}\right)} \leq C \sum_{j=1}^{N}\left\|\frac{\partial f_{i}\left(y^{\prime}, y_{n}, u_{1}^{\lambda}, \ldots, u_{j}^{\lambda}+\theta_{j} w_{j}^{\lambda}, \ldots, u_{N}\right)}{\partial u_{j}} w_{j}^{\lambda}\right\|_{L^{n r /(n+2 m r)}\left(\Sigma_{\lambda}^{j}\right)},
$$

where $0<\theta_{j}<1$. Then, from Hölder's inequality, we have $\left\|w_{i}^{\lambda}\right\|_{L^{r}\left(\Sigma_{\lambda}^{i}\right)} \leq C \sum_{j=1}^{N}\left\|\frac{\partial f_{i}\left(y^{\prime}, y_{n}, u_{1}^{\lambda}, \ldots, u_{j}^{\lambda}+\theta_{j} w_{j}^{\lambda}, \ldots, u_{N}\right)}{\partial u_{j}}\right\|_{L^{n /(2 m)}\left(\Sigma_{\lambda}^{j}\right)}\left\|w_{j}^{\lambda}\right\|_{L^{r}\left(\Sigma_{\lambda}^{j}\right)}$.

Moreover, taking the sum from $i$ to $N$ gives

$$
\sum_{i=1}^{N}\left\|w_{i}^{\lambda}\right\|_{L^{r}\left(\Sigma_{\lambda}^{i}\right)} \leq C \sum_{i=1}^{N} \sum_{j=1}^{N}\left\|\frac{\partial f_{i}}{\partial u_{j}}\right\|_{L^{n /(2 m)}\left(\Sigma_{\lambda}^{j}\right)}\left\|w_{j}^{\lambda}\right\|_{L^{r}\left(\Sigma_{\lambda}^{j}\right)}
$$

By virtue of the properties $\left(f_{i}^{1}\right)$ and $\left(f_{i}^{2}\right)$ of the functions $f_{i}$, we can choose $\lambda$ negative enough that

$$
C \sum_{i=1}^{N} \sum_{j=1}^{N}\left\|\frac{\partial f_{i}}{\partial u_{j}}\right\|_{L^{n /(2 m)}\left(\Sigma_{\lambda}^{j}\right)} \leq \frac{1}{2}
$$

This implies that

$$
\sum_{i=1}^{N}\left\|w_{i}^{\lambda}\right\|_{L^{r}}=0 .
$$

Therefore, $\Sigma_{i}^{\lambda}$ must be of measure zero and $u_{i}(x) \leq u_{i}^{\lambda}(x)$ a.e. in $\Sigma_{\lambda}$ for every $1 \leq i<n$. 
Step 2: We will prove that the plane can be moved to a critical point

$$
\lambda_{0}=\sup \left\{\lambda \mid u_{i}(x) \leq u_{i}^{\lambda}(x),-\infty<\lambda<0, x \in \Sigma_{\lambda}, i=1, \ldots, N\right\}
$$

such that $w_{i}^{\lambda_{0}} \equiv 0$.

If $\lambda_{0}=0$, move the plane from the positive infinity to the origin and argue as before. Hence assume $\lambda_{0}<0$, so obviously $u_{i}(x) \leq u_{i}^{\lambda_{0}}(x)$. We claim that

$$
u_{i}(x)<u_{i}^{\lambda_{0}}(x) \quad \text { or } \quad u_{i}(x) \equiv u^{\lambda_{0}}(x)
$$

in $\Sigma_{\lambda_{0}}$ for $i=1, \ldots, N$. If not, then there exists some $i \in\left\{i_{1}, \ldots, i_{l_{0}}\right\} \subset\{1, \ldots, N\}$ such that $u_{i}\left(x^{0}\right)=u_{i}^{\lambda_{0}}\left(x^{0}\right)$ for some $x^{0} \in \Sigma_{\lambda_{0}}$, but $u_{i}(x) \not \equiv u_{i}^{\lambda_{0}}(x)$. Therefore $u_{i}(x)<u_{i}^{\lambda_{0}}(x)$ for $i \in S=\{1, \ldots, N\} \backslash\left\{i_{1}, \ldots, i_{l 0}\right\}$ for any $x \in \Sigma_{\lambda_{0}}$. Now

$$
\begin{aligned}
& w_{i}^{\lambda}\left(x^{0}\right) \\
& \leq \int_{\Sigma_{\lambda}}\left(G\left(x_{\lambda}^{0}, y_{\lambda}\right)-G\left(x^{0}, y_{\lambda}\right)\right)\left(f_{i}\left(y^{\prime}, y_{n}, u_{1}, \ldots, u_{N}\right)-f_{i}\left(y^{\prime}, y_{n}, u_{1}^{\lambda}, \ldots, u_{N}^{\lambda}\right)\right) \\
& \leq 0
\end{aligned}
$$

thus, $f_{i}\left(y^{\prime}, y_{n}, u_{1}, \ldots, u_{N}\right) \equiv f_{i}\left(y^{\prime}, y_{n}, u_{1}^{\lambda}, \ldots, u_{N}^{\lambda}\right)$ for $i \in\left\{i_{1}, \ldots, i_{l 0}\right\}$. Hence

$$
\sum_{i=i_{1}}^{i_{l_{0}}} f_{i}\left(y^{\prime}, y_{n}, u_{1}, \ldots, u_{N}\right) \equiv \sum_{i=i_{1}}^{i_{l_{0}}} f_{i}\left(y^{\prime}, y_{n}, u_{1}^{\lambda}, \ldots, u_{N}^{\lambda}\right) .
$$

This contradicts the assumption that $f_{i}$ are essentially related, which implies the claim is true.

Suppose $u_{i}(x)<u_{i}^{\lambda_{0}}(x)$ in $\Sigma_{\lambda_{0}}$ for $i=1, \ldots, N$. In the spirit of Lemma 4, we will show that the plane can move to the right a little bit further. By the assumption $\left(f_{i}^{2}\right)$, for any small $\epsilon$, there exists a large enough ball $\mathbb{B}_{R}(0)$ such that

$$
\left\|\frac{\partial f_{i}}{\partial u_{j}}\right\|_{L^{n /(2 m)}\left(\mathbb{R}_{+}^{n} \backslash \mathbb{B}_{R}\right)}<\epsilon,
$$

for $i, j=1, \ldots, N$. From Lusin's theorem, for any $\delta$, there exists a closed set $F_{\delta}$ such that $\left.w_{i}^{\lambda_{0}}\right|_{F_{\delta}}$ is continuous for $i=1, \ldots, N$, with $F_{\delta} \subset E:=\mathbb{B}_{R}(0) \cap \Sigma_{\lambda_{0}}$ and $m\left(E-F_{\delta}\right)<\delta$. As $w_{i}^{\lambda_{0}}(x)<0$ in the interior of $\Sigma_{\lambda_{0}}, w^{\lambda_{0}}(x)<0$ in $F_{\delta}$. Choosing $\epsilon_{1}$ sufficiently small gives that for any $\lambda \in\left[\lambda_{0}, \lambda_{0}+\epsilon_{1}\right)$,

$$
w_{i}^{\lambda}<0 \quad \text { for all } x \in F_{\delta}
$$

by continuity. It follows that for such $\lambda$,

$$
\Sigma_{\lambda}^{i} \subset M:=\left(\mathbb{R}_{+}^{n} \backslash \mathbb{B}_{R}(0)\right) \cup\left(E \backslash F_{\delta}\right) \cup\left(\left(\Sigma_{\lambda} \backslash \Sigma_{\lambda_{0}}^{-}\right) \cap \mathbb{B}_{R}(0)\right)
$$


for $i=1, \ldots, N$. Choosing $\epsilon, \delta$ and $\epsilon_{1}$ small enough and using absolute continuity of integration, we conclude that

$$
C \sum_{i=1}^{N} \sum_{j=1}^{N}\left\|\frac{\partial f_{i}}{\partial u_{j}}\right\|_{L^{n /(2 m)}(M)} \leq \frac{1}{2} .
$$

Then, similarly to (22), $\sum_{i=1}^{N}\left\|w_{i}^{\lambda}\right\|_{L^{q}}=0$. Therefore, $\Sigma_{\lambda}^{i}$ must be of measure zero, which contradicts the definition of $\lambda_{0}$. Hence $w_{i}^{\lambda_{0}} \equiv 0$ in $\Sigma_{\lambda_{0}}$ for $i=1, \ldots, N$.

For the $x_{n}$-coordinate, start moving the plane from $x_{n}=0$ to positive infinity. If $\lambda>0$ is sufficiently small, then we can show as in Step 1 that $w_{i}^{\lambda}(x)<0$.

Next, we prove that, if $w_{i}^{\lambda}(x) \not \equiv 0$, then

$$
\lambda_{0}=\sup _{\lambda}\left\{u_{i}^{\lambda}(x)>u(x), \lambda>0, x \in \Sigma_{\lambda}, i=1, \ldots, N\right\}=\infty .
$$

If not, then $\lambda_{0}<\infty$. It is also known that $w_{i}^{\lambda_{0}}<0$ or $w_{i}^{\lambda_{0}} \equiv 0$ for $x \in \Sigma_{\lambda_{0}}$ for $i=1, \ldots, N$. Hence $w_{i}^{\lambda}<0$ for any $\lambda$. If $w_{i}^{\lambda}(x) \equiv 0$, since $u_{i}\left(x^{\prime}, x_{n}\right)=0$ on $x_{n}=0$, then $u_{i}(x) \equiv 0$. Therefore, $u_{i}(x)$ is nondecreasing in the $x_{n}$ direction for every $i$.

Proof of Theorem 2. By Lemma 5, $u_{i}(x)$ is nondecreasing in the $x_{n}$ direction. By the assumption that $u \in L^{r}\left(\mathbb{R}_{+}^{n}\right)$ for some $r>n /(n-2 m)$, for any $a \in \mathbb{R}_{+}$, then

$$
\int_{\mathbb{R}_{+}^{n}}\left|u_{i}\left(x^{\prime}, x_{n}\right)\right|^{r} d x^{\prime} d x_{n} \geq \int_{\mathbb{R}^{n-1}} \int_{a}^{\infty}\left|u_{i}\left(x^{\prime}, a\right)\right|^{r} d x^{\prime} d x_{n} .
$$

The integrability of nonnegative $u_{i}$ implies $u_{i}\left(x^{\prime}, a\right)=0$ for any $a$ and $x^{\prime} \in \mathbb{R}^{n-1}$. Thus $u_{i}(x)=0$ in $\mathbb{R}_{+}^{n}$ for $i=1, \ldots, N$.

\section{Proof of Theorem 3}

In this section, we prove the regularity of the solutions in (6) which is related to the Dirichlet problems of polyharmonic elliptic equations. For the convenience of the reader, we present a regularity lifting lemma (Lemma 6), initially used in [Chen and $\mathrm{Li} 2010]$.

Let $Z$ be a given vector space. Let $\|\cdot\|_{X}$ and $\|\cdot\|_{Y}$ be two norms on $Z$. Define a new norm $\|\cdot\|_{Z}$ by

$$
\|\cdot\|_{Z}=\sqrt[p]{\|\cdot\|_{X}^{p}+\|\cdot\|_{Y}^{p}} .
$$

For simplicity, we assume that $Z$ is complete with respect to the norm $\|\cdot\|_{Z}$. Let $X$ and $Y$ be the completions of $Z$ under $\|\cdot\|_{X}$ and $\|\cdot\|_{Y}$, respectively. Here $p$ can be chosen between 1 and $\infty$, according to need. It is easy to see that $Z=X \cap Y$.

Lemma 6 (regularity lifting lemma). Let $T$ be a contraction map from $X$ into itself and from $Y$ into itself. Assume that $f \in X$, and that there exists a function $g \in Z$ such that $f=T f+g$. Then $f$ also belongs to $Z$. 
Proof of Theorem 3. From the integral representation of $G(x, y)$, we have the estimates

$$
|G(x, y)| \leq C|x-y|^{2 m-n}
$$

Define the linear operator

$$
T_{u} w(x)=\int_{\mathbb{R}_{+}^{n}} G(x, y)|u|^{p-1} w d y .
$$

For any real number $a>0$, define

$$
\begin{cases}u_{a}(x)=u(x) & \text { if }|u(x)|>a \text { or }|x|>a \\ u_{a}(x)=0 & \text { otherwise. }\end{cases}
$$

Let $u_{b}(x)=u(x)-u_{a}(x)$. Since $u$ satisfies (6), it follows that

$$
u_{a}(x)=\int_{\mathbb{R}_{+}^{n}} G(x, y)\left|u_{a}\right|^{p-1} u_{a} d y+I(x),
$$

where

$$
I(x)=\int_{\mathbb{R}_{+}^{n}} G(x, y)\left|u_{b}\right|^{p-1} u_{b} d y-u_{b}(x) .
$$

We claim that for any $r>n /(n-2 m)$

$$
I(x) \in L^{\infty}\left(\mathbb{R}_{+}^{n}\right) \cap L^{r}\left(\mathbb{R}_{+}^{n}\right) .
$$

In order to prove this claim, by the definition of $u_{b}$, we only need to show that

$$
B(x):=\int_{\mathbb{R}_{+}^{n}} G(x, y)\left|u_{b}\right|^{p-1} u_{b} d y \in L^{\infty}\left(\mathbb{R}_{+}^{n}\right) \cap L^{r}\left(\mathbb{R}_{+}^{n}\right) .
$$

By (23),

$$
|B(x)| \leq C \int_{\mathbb{R}_{+}^{n}}|x-y|^{2 m-n}\left|u_{b}\right|^{p} d y .
$$

Applying the Hardy-Littlewood-Sobolev inequality to (27) gives that for any $r>$ $n /(n-2 m)$,

$$
\|B(x)\|_{L^{r}} \leq C\left\|u_{b}\right\|_{L^{n r /(n+2 m r)}}<\infty
$$

by the definition of $u_{b}$.

For any $x \in \mathbb{B}_{2 a}$, we estimate that

$$
|B(x)| \leq C \int_{\mathbb{R}_{+}^{n} \cap \mathbb{B}_{a}}|x-y|^{2 m-n} d y<\infty,
$$


while for any $x \notin B_{2 a}$,

$$
|B(x)| \leq C \int_{\mathbb{R}_{+}^{n} \cap \mathbb{B}_{a}}|x-y|^{2 m-n} d y \leq C a^{2 m-n} \int_{\mathbb{R}_{+}^{n} \cap \mathbb{B}_{a}} d y<\infty .
$$

Combining this inequality with (29) and (28), we conclude that

$$
B(x) \in L^{\infty}\left(\mathbb{R}_{+}^{n}\right) \cap L^{r}\left(\mathbb{R}_{+}^{n}\right) .
$$

Therefore, we have proved the claim.

Next, we prove $T_{u_{a}}$ is a contraction on $L^{s}\left(\mathbb{R}_{+}^{n}\right)$ for any $s>n /(n-2 m)$. By (23), for any $s>n /(n-2 m)$,

$$
\left\|T_{u_{a}} w\right\|_{L^{s}\left(\mathbb{R}_{+}^{n}\right)} \leq C\left\|\int_{\mathbb{R}_{+}^{n}}|x-y|^{2 m-n}\left|u_{a}(y)\right|^{p-1} w(y) d y\right\|_{L^{s}\left(\mathbb{R}_{+}^{n}\right)} .
$$

Using the Hardy-Littlewood-Sobolev inequality, then Hölder's inequality gives

$$
\left\|T_{u_{a}} w\right\|_{L^{s}\left(\mathbb{R}_{+}^{n}\right)} \leq C\left\|\left|u_{a}\right|^{(p-1)}\right\|_{L^{n} /(2 m)\left(\mathbb{R}_{+}^{n}\right)}\|w\|_{L^{s}\left(\mathbb{R}_{+}^{n}\right)} .
$$

Since $u(x) \in L^{(p-1) n /(2 m)}\left(\mathbb{R}_{+}^{n}\right)$, choosing $a$ large enough, we have

$$
\left\|T_{u_{a}} w\right\|_{L^{s}\left(\mathbb{R}_{+}^{n}\right)} \leq \frac{1}{2}\|w\|_{L^{s}\left(\mathbb{R}_{+}^{n}\right)} .
$$

Therefore $T_{u_{a}}$ is a contraction map on $L^{s}\left(\mathbb{R}_{+}^{n}\right)$ for any $s>n /(n-2 m)$ when $a$ is sufficiently large. Applying (30) to the case of $s=q=(p-1) n /(2 m)$ which is greater than $n /(n-2 m)$ when $p>n /(n-2 m)$ and to the case of $s>n /(n-2 m)$, the regularity lifting lemma implies that the unique solution $u_{a}$ is in $L^{q} \cap L^{s}$, which means $u \in L^{q} \cap L^{s}$ for any $s>n /(n-2 m)$.

Finally, we claim that $u \in L^{\infty}\left(\mathbb{R}_{+}^{n}\right)$.

As in (25) and the definition of $u_{a}$, it suffices to prove that

$$
A(x):=\int_{\mathbb{R}_{+}^{n}} G(x, y)\left|u_{a}\right|^{p-1} u_{a} d y \in L^{\infty} .
$$

For any $x \in \mathbb{R}_{+}^{n}$, by (23),

$$
|A(x)| \leq C \int_{\mathbb{R}_{+}^{n} \cap \mathbb{B}_{a}(x)}|x-y|^{2 m-n}\left|u_{a}\right|^{p} d y+C \int_{\mathbb{R}_{+}^{n} \backslash \mathbb{B}_{a}(x)}|x-y|^{2 m-n}\left|u_{a}\right|^{p} d y .
$$

Using Hölder's inequality and the property that $u \in L^{q} \cap L^{s}$ for any $s>n /(n-2 m)$, respectively, we obtain, for any fixed $a$,

$$
\begin{aligned}
& \int_{\mathbb{R}_{+}^{n} \cap \mathbb{B}_{2 a}(x)}|x-y|^{2 m-n}\left|u_{a}\right|^{p} d y<\infty, \\
& \int_{\mathbb{R}_{+}^{n} \backslash \mathbb{B}_{2 a}(x)}|x-y|^{2 m-n}\left|u_{a}\right|^{p} d y<\infty .
\end{aligned}
$$


These estimates imply that $A(x)<\infty$, therefore $u \in L^{\infty}\left(\mathbb{R}_{+}^{n}\right)$, so Theorem 3 holds. (Actually, we have proved that $L^{s}\left(\mathbb{R}_{+}^{n}\right) \cap L^{\infty}\left(\mathbb{R}_{+}^{n}\right)$ for any $s>n /(n-2 m)$.)

Proof of Corollary 2. From the proof of Theorem 3, we have that $u(x) \in L^{q} \cap L^{s_{0}}$ for any $s_{0}>n /(n-2 m)$, where $q=(p-1) n /(2 m)$. Then $u \in L^{2 n /(n-2 m)}$. Let $f(u)=u^{p}$ in (1). Obviously the assumptions of $f$ in Theorem 1 are satisfied. Therefore, $u$ is trivial in (7).

\section{Proof of Theorem 4}

In this section, we sketch the proof of Theorem 4. For a complete proof, refer to the proofs of Theorems 1 and 2. Let

$$
v(x)=\frac{1}{|x|^{n-2 m}} u\left(\frac{x}{|x|^{2}}\right)
$$

be the Kelvin transform of $u(x)$ centered at the origin. Then $v(x)$ solves

$$
v(x)=\int_{\mathbb{R}_{+}^{n}} G(x, y)|y|^{(n-2 m) p-(n+2 m)} v^{p}(y) d y .
$$

Since $u \in L_{\text {loc }}^{2 n /(n-2 m)}\left(\mathbb{R}_{+}^{n}\right)$, then $v \in L^{2 n /(n-2 m)}\left(\Omega^{\prime}\right)$, where $\Omega^{\prime}$ is an arbitrary domain in $\mathbb{R}_{+}^{n}$ with $\operatorname{dist}\left(\Omega^{\prime}, 0\right)>d>0$ for some positive $d$. Moreover, $v \in L^{(p-1) n /(2 m)}\left(\Omega^{\prime}\right)$, since $1<p<(n+2 m) /(n-2 m)$. As in the proofs of Lemma 2 and Theorems 1 and 2 , for $1 \leq i<n$,

$$
\begin{aligned}
& v(x)-v\left(x_{\lambda}\right) \\
& =\int_{\Sigma_{\lambda}}\left(G\left(x_{\lambda}, y_{\lambda}\right)-G\left(x, y_{\lambda}\right)\right)\left(|y|^{(n-2 m) p-(n+2 m)} v^{p}-\left|y^{\lambda}\right|^{(n-2 m) p-(n+2 m)} v_{\lambda}^{p}\right) d y \\
& \leq \int_{\Sigma_{\lambda}}\left(G\left(x_{\lambda}, y_{\lambda}\right)-G\left(x, y_{\lambda}\right)\right)|y|^{(n-2 m) p-(n+2 m)}\left(v^{p}-v_{\lambda}^{p}\right) d y \\
& \leq C|\lambda|^{(n-2 m) p-(n+2 m)} \int_{\Sigma_{\lambda}^{-}} G\left(x_{\lambda}, y_{\lambda}\right)\left(v^{p}-v_{\lambda}^{p}\right) d y .
\end{aligned}
$$

Following the steps in proving the axial symmetry of $u$ in Theorems 1 and 2, we can show that $v(x)$ is axially symmetric with respect to $x_{n}$ axis. Let $\left(x^{1}, x_{n}\right)$ and $\left(x^{2}, x_{n}\right)$ be two points in $\mathbb{R}_{+}^{n}$, where $x^{1}, x^{2}$ are arbitrary in $\mathbb{R}^{n-1}$. Let $x^{0}$ be the midpoint of the line segment $\overline{x^{1} x^{2}}$. Consider the Kelvin transform of $u(x)$ centered at $x^{*}=\left(x^{0}, 0\right)$, that is,

$$
v(x)=\frac{1}{\left|x-x^{*}\right|^{n-2 m}} u\left(\frac{x-x^{*}}{\left|x-x^{*}\right|^{2}}\right) .
$$

Then $v(x)$ is axially symmetric with respect to $x^{\prime}=x^{0}$. In particular, $u\left(x^{1}, x_{n}\right)=$ $u\left(x^{2}, x_{n}\right)$. Since $x^{1}$ and $x^{2}$ are any two points in $\mathbb{R}^{n-1}$, the function $u\left(x^{\prime}, x_{n}\right)$ is constant for any fixed $x_{n}$. Therefore, $u(x)$ only depends on $x_{n}$. 


\section{Acknowledgement}

The authors wish to thank the referee for his very careful reading and constructive suggestions.

\section{References}

[Bachar et al. 2004] I. Bachar, H. Mâagli, and M. Zribi, "Estimates on the Green function and existence of positive solutions for some polyharmonic nonlinear equations in the half space", Manuscripta Math. 113:3 (2004), 269-291. MR 2006i:35063 Zbl 1065.35108

[Berchio et al. 2008] E. Berchio, F. Gazzola, and T. Weth, "Radial symmetry of positive solutions to nonlinear polyharmonic Dirichlet problems", J. Reine Angew. Math. 620 (2008), 165-183. MR 2009i:35069 Zbl 1182.35109

[Boggio 1905] T. Boggio, "Sulle funzioni di Green d'ordine m", Rend. Circ. Mat. Palermo 20 (1905), 97-135. JFM 36.0827.01

[Caffarelli et al. 1989] L. A. Caffarelli, B. Gidas, and J. Spruck, "Asymptotic symmetry and local behavior of semilinear elliptic equations with critical Sobolev growth", Comm. Pure Appl. Math. 42:3 (1989), 271-297. MR 90c:35075 Zbl 0702.35085

[Chang and Yang 1997] S.-Y. A. Chang and P. C. Yang, "On uniqueness of solutions of $n$th order differential equations in conformal geometry", Math. Res. Lett. 4:1 (1997), 91-102. MR 97m:58204 Zbl 0903.53027

[Chen and Li 1991] W. Chen and C. Li, "Classification of solutions of some nonlinear elliptic equations", Duke Math. J. 63:3 (1991), 615-622. MR 93e:35009 Zbl 0768.35025

[Chen and Li 2010] W. Chen and C. Li, Methods on nonlinear elliptic equations, AIMS Series on Differential Equations \& Dynamical Systems 4, American Insitute of Mathematical Sciences, Springfield, MO, 2010. MR 2759774 Zbl 1214.35023

[Chen and Zhu 2011] W. Chen and J. Zhu, "Radial symmetry and regularity of solutions for polyharmonic Dirichlet problems", J. Math. Anal. Appl. 377:2 (2011), 744-753. MR 2769171 Zbl 1211.35101

[Chen et al. 2005] W. Chen, C. Li, and B. Ou, "Qualitative properties of solutions for an integral equation”, Discrete Contin. Dyn. Syst. 12:2 (2005), 347-354. MR 2006g:45009 Zbl 1081.45003

[Chen et al. 2006] W. Chen, C. Li, and B. Ou, "Classification of solutions for an integral equation", Comm. Pure Appl. Math. 59:3 (2006), 330-343. MR 2006m:45007a Zbl 1093.45001

[Gidas et al. 1979] B. Gidas, W. M. Ni, and L. Nirenberg, "Symmetry and related properties via the maximum principle", Comm. Math. Phys. 68:3 (1979), 209-243. MR 80h:35043 Zbl 0425.35020

[Hang 2007] F. Hang, "On the integral systems related to Hardy-Littlewood-Sobolev inequality", Math. Res. Lett. 14:3 (2007), 373-383. MR 2008j:26037 Zbl 1144.26031

[Jin and Li 2006] C. Jin and C. Li, "Symmetry of solutions to some systems of integral equations", Proc. Amer. Math. Soc. 134:6 (2006), 1661-1670. MR 2006j:45017 Zbl 1156.45300

[Li 2004] Y. Y. Li, "Remark on some conformally invariant integral equations: the method of moving spheres”, J. Eur. Math. Soc. (JEMS) 6:2 (2004), 153-180. MR 2005e:45007 Zbl 1075.45006

[Li and Ma 2008] C. Li and L. Ma, "Uniqueness of positive bound states to Schrödinger systems with critical exponents", SIAM J. Math. Anal. 40:3 (2008), 1049-1057. MR 2009k:35079 Zbl 1167.35347

[Ma and Chen 2006] L. Ma and D. Chen, "A Liouville type theorem for an integral system", Commun. Pure Appl. Anal. 5:4 (2006), 855-859. MR 2007d:35094 Zbl 1134.45007 
[Ma and Chen 2008] L. Ma and D. Chen, "Radial symmetry and monotonicity for an integral equation”, J. Math. Anal. Appl. 342:2 (2008), 943-949. MR 2009m:35151 Zbl 1140.45004

[Qing and Raske 2006] J. Qing and D. Raske, "On positive solutions to semilinear conformally invariant equations on locally conformally flat manifolds", Int. Math. Res. Not. 2006 (2006), Art. ID 94172. MR 2007a:58044 Zbl 1115.53028

[Reichel and Weth 2009] W. Reichel and T. Weth, "A priori bounds and a Liouville theorem on a half-space for higher-order elliptic Dirichlet problems", Math. Z. 261:4 (2009), 805-827. MR 2010b:35088 Zbl 1167.35014

[Serrin 1971] J. Serrin, "A symmetry problem in potential theory", Arch. Rational Mech. Anal. 43 (1971), 304-318. MR 48 \#11545 Zbl 0222.31007

[Stein 1970] E. M. Stein, Singular integrals and differentiability properties of functions, Princeton Mathematical Series 30, Princeton University Press, 1970. MR 44 \#7280 Zbl 0207.13501

[Wei and Xu 1999] J. Wei and X. Xu, "Classification of solutions of higher order conformally invariant equations”, Math. Ann. 313:2 (1999), 207-228. MR 2000a:58093 Zbl 0940.35082

Received September 9, 2010. Revised May 30, 2011.

GUOZHEN LU

DEPARTMENT OF MATHEMATICS

WAYNE STATE UNIVERSITY

DETROIT MI 48202

UNITED STATES

gzlu@math.wayne.edu

http://www.math.wayne.edu/ gzlu

JIUYI ZHU

DEPARTMENT OF MATHEMATICS

WAYNE STATE UNIVERSITY

DETROIT MI 48202

UNITED STATES

jiuyi.zhu@wayne.edu 


\title{
PACIFIC JOURNAL OF MATHEMATICS
}

\author{
http://pacificmath.org \\ Founded in 1951 by \\ E. F. Beckenbach (1906-1982) and F. Wolf (1904-1989)
}

\section{EDITORS}

V. S. Varadarajan (Managing Editor)

Department of Mathematics

University of California

Los Angeles, CA 90095-1555

pacific@math.ucla.edu

Vyjayanthi Chari

Department of Mathematics

University of California

Riverside, CA 92521-0135

chari@math.ucr.edu

\section{Robert Finn}

Department of Mathematics Stanford University

Stanford, CA 94305-2125

finn@math.stanford.edu

Kefeng Liu

Department of Mathematics

University of California

Los Angeles, CA 90095-1555

liu@math.ucla.edu
Darren Long

Department of Mathematics

University of California

Santa Barbara, CA 93106-3080

long@math.ucsb.edu

Jiang-Hua Lu

Department of Mathematics

The University of Hong Kong

Pokfulam Rd., Hong Kong jhlu@maths.hku.hk

Alexander Merkurjev

Department of Mathematics

University of California

Los Angeles, CA 90095-1555

merkurev@math.ucla.edu
Sorin Popa

Department of Mathematics University of California

Los Angeles, CA 90095-1555 popa@math.ucla.edu

Jie Qing

Department of Mathematics

University of California

Santa Cruz, CA 95064

qing@cats.ucsc.edu

Jonathan Rogawski

Department of Mathematics

University of California

Los Angeles, CA 90095-1555

jonr@math.ucla.edu

\section{PRODUCTION}

pacific@math.berkeley.edu

\section{SUPPORTING INSTITUTIONS}

ACADEMIA SINICA, TAIPEI

CALIFORNIA INST. OF TECHNOLOGY INST. DE MATEMÁTICA PURA E APLICADA KEIO UNIVERSITY

MATH. SCIENCES RESEARCH INSTITUTE NEW MEXICO STATE UNIV.

OREGON STATE UNIV.

\author{
STANFORD UNIVERSITY \\ UNIV. OF BRITISH COLUMBIA \\ UNIV. OF CALIFORNIA, BERKELEY \\ UNIV. OF CALIFORNIA, DAVIS \\ UNIV. OF CALIFORNIA, LOS ANGELES \\ UNIV. OF CALIFORNIA, RIVERSIDE \\ UNIV. OF CALIFORNIA, SAN DIEGO \\ UNIV. OF CALIF., SANTA BARBARA
}

\author{
UNIV. OF CALIF., SANTA CRUZ \\ UNIV. OF MONTANA \\ UNIV. OF OREGON \\ UNIV. OF SOUTHERN CALIFORNIA \\ UNIV. OF UTAH \\ UNIV. OF WASHINGTON \\ WASHINGTON STATE UNIVERSITY
}

These supporting institutions contribute to the cost of publication of this Journal, but they are not owners or publishers and have no responsibility for its contents or policies.

See inside back cover or pacificmath.org for submission instructions.

The subscription price for 2011 is US \$420/year for the electronic version, and \$485/year for print and electronic.

Subscriptions, requests for back issues from the last three years and changes of subscribers address should be sent to Pacific Journal of Mathematics, P.O. Box 4163, Berkeley, CA 94704-0163, U.S.A. Prior back issues are obtainable from Periodicals Service Company, 11 Main Street, Germantown, NY 12526-5635. The Pacific Journal of Mathematics is indexed by Mathematical Reviews, Zentralblatt MATH, PASCAL CNRS Index, Referativnyi Zhurnal, Current Mathematical Publications and the Science Citation Index.

The Pacific Journal of Mathematics (ISSN 0030-8730) at the University of California, c/o Department of Mathematics, 969 Evans Hall, Berkeley, CA 94720-3840, is published monthly except July and August. Periodical rate postage paid at Berkeley, CA 94704, and additional mailing offices. POSTMASTER: send address changes to Pacific Journal of Mathematics, P.O. Box 4163, Berkeley, CA 94704-0163.

PJM peer review and production are managed by EditFLOW ${ }^{\mathrm{TM}}$ from Mathematical Sciences Publishers.

PUBLISHED BY PACIFIC JOURNAL OF MATHEMATICS

at the University of California, Berkeley 94720-3840

A NON-PROFIT CORPORATION

Typeset in LATEX

Copyright $(2011$ by Pacific Journal of Mathematics 


\section{PACIFIC JOURNAL OF MATHEMATICS}

Volume $253 \quad$ No. $2 \quad$ October 2011

Fusion rules on a parametrized series of graphs

MARTA AsAeda and UfFE HAAGERUP

Group gradings on restricted Cartan-type Lie algebras

YURI BAHTURIN and MIKHAIL KOCHETOV

B2-convexity implies strong and weak lower semicontinuity of partitions of $\mathbb{R}^{n}$

\section{DAVID G. CARABALLO}

Testing the functional equation of a high-degree Euler product

DAVID W. FARMER, NATHAN C. RYAN and RALF SCHMIDT

Asymptotic structure of a Leray solution to the Navier-Stokes flow around a 367 rotating body

Reinhard Farwig, Giovanni P. Galdi and Mads Kyed

Type II almost-homogeneous manifolds of cohomogeneity one

DANIEL GUAN

Cell decompositions of Teichmüller spaces of surfaces with boundary

REN GUO and FENG LUO

A system of third-order differential operators conformally invariant under $\mathfrak{s l}(3, \mathbb{C})$ and $\mathfrak{s o}(8, \mathbb{C})$

Toshinis A KUBO

Axial symmetry and regularity of solutions to an integral equation in a half-space

GUOZHEN LU and JIUYi ZHU

Braiding knots in contact 3-manifolds

\section{ELENA PAVELESCU}

Gradient estimates for positive solutions of the heat equation under geometric flow

JUN SUN 